Revista de Psicología y Educación / Journal of Psychology and Education, 2019, 14(1), 74-86 (www.rpye.es) Doi: https://doi.org/10.23923/rpye2019.01.173

ISSN: $1699-9517$

\title{
Capacidad metacognitiva en docentes y futuros docentes de enseñanza no universitaria

\author{
Ruth Pinedo-González ${ }^{\star 1}$, Manuel Cañas-Encinas' , Noelia García-Martín’ y \\ Noelia García-González²
}

'Universidad de Valladolid

2 EOEP Alcobendas

\begin{abstract}
Resumen: La capacidad metacognitiva es una competencia docente clave para instaurar el Aprendizaje Basado en el Pensamiento en las aulas. El objetivo de este estudio es desvelar las concepciones que los docentes tienen sobre el pensamiento. Se han analizado los mapas conceptuales realizados por 416 docentes y futuros docentes en los cuales daban respuesta a estas preguntas: ¿̇qué es el pensamiento?; cuando dices a alguien que estás pensando żqué tipo de cosas podrían estar pasando realmente en tu cabeza? Las respuestas se categorizaron en propias del pensamiento estratégico y no estratégico, así como en diferentes subtipos dentro de ambas categorías. Los datos fueron analizados de forma descriptiva e inferencial. Los resultados revelan el predominio del pensamiento estratégico. Los profesores en activo tienen mayor porcentaje de respuestas de pensamiento estratégico que los futuros docentes. Los docentes en formación presentan diferencias significativas en estrategias y subestrategias de pensamiento según el curso y la titulación que estudian y los docentes en activo según la etapa educativa en la que ejercen. Estos datos apoyan la idea de que enriquecer los programas de formación inicial y permanente del profesorado con herramientas metacognitivas puede contribuir a un posterior trabajo más sistemático del pensamiento con su alumnado.
\end{abstract}

Palabras clave: Aprendizaje Basado en el Pensamiento, Estrategias de Pensamiento, Formación del Profesorado, Mapas Conceptuales, Metacognición.

\begin{abstract}
Meta-strategic knowledge in teachers and pre-service teachers of non-university education
Abstract: Meta-strategic knowledge is a key teaching competence to establish Thinking-Based Learning in classroom. The objective of this study is uncovering teachers' thinking about thinking. Concept maps from 416 teachers and pre-service teachers were analyzed. The sample developed a concept-map to answer the following question: "What is thinking? When you tell someone you are thinking, what kind of things might actually be going on in your head?" Answers were categorized as strategic and non-strategic thinking, as well as to different subtypes within both categories. Results revealed the predominance of strategic thinking. Teachers have more strategic thinking responses than pre-service teachers. Pre-service teachers present significant differences according to the course and the degree that they study and teachers according to the educative stage in which they exert. These data support the idea that enriching initial and ongoing teacher training programs with metacognitive tools can contribute to a systematic work of student thinking.

Keywords: Conceptual Maps, Metacognition, Teacher Training, Thinking-Based Learning, Thinking Strategies.
\end{abstract}

El S. XXI trae consigo una revolución en el aprendizaje. Las metodologías tradicionales que implican aprendizajes memorísticos y superficiales ya no son válidas para un

Recibido: 28/09/2018 - Aceptado: 29/1 1/2018 - Avance online: 27/12/2018 *Correspondencia: Ruth Pinedo González.

Universidad de Valladolid

C.P: 40005, Segovia, España.

E-mail: ruth.pinedo@uva.es alumnado nativo de la sociedad de la información, nacido en plena modernidad líquida (Bauman, 2006). Estas metodologías tradicionales, fundamentadas en la transmisiónrecepción, todavía siguen presentes en las aulas como consecuencia, entre otras muchas causas, de las exigencias curriculares y las pruebas estandarizadas (Labrador y Andreu, 2008). 
Tal y como sostienen Pinedo, Caballero y Fernández (2016), se requiere de metodologías activas que generen en los estudiantes aprendizajes significativos, duraderos y transferibles a otros contextos. Se ha de buscar que el alumnado de uso a los conocimientos que adquieren en el proceso de enseñanza (Perkins, 2008). Por ello, el centro de interés se pone en la comprensión del contenido y en el desarrollo de un pensamiento profundo que les permita aprender de forma autónoma y eficaz (Swartz, Costa, Beyer y Reagan y Kallick, 2013).

En esa línea, el proceso de enseñanzaaprendizaje ha de asegurar que el alumnado, progresivamente, adquiera la competencia aprender a aprender (Orden ECD/65/2015), la cual engloba habilidades como: iniciar, organizar y persistir en el aprendizaje; organizar y gestionar el aprendizaje; conocer y controlar los propios procesos de aprendizaje para ajustarlos a los tiempos y las demandas de las tareas y actividades que conducen al aprendizaje y reflexionar y tomar conciencia de los propios procesos mentales implicados en dicho aprendizaje.

Para alcanzar estas habilidades son necesarias destrezas reflexivas y de autorregulación, tales como: estrategias de planificación, plan de acción, estrategias de supervisión y evaluación. En otras palabras, lo que Swartz et al. (2017) llaman autonomía reflexiva: "ser conscientes del proceso de pensamiento que estamos llevando a cabo, planificarlo, ejecutarlo reflexionando sobre lo que estamos haciendo y evaluar el resultado" (p. 104).

Este cambio en el foco de atención del proceso educativo implica la adquisición de nuevas competencias docentes, tanto en la formación inicial como permanente, para que los estudiantes tomen conciencia de los procesos de pensamiento. Así pues, siguiendo lo expuesto en la OECD (2001) desde una perspectiva educativa, se pueden señalar competencias docentes como: habilidad para aprender, resolución de problemas y habilidades analíticas. Entre otras, estas implican: dominar los contenidos; tener como metas los hábitos de estudio, la metacognición y la capacidad de mediar para que su alumnado desarrolle las habilidades que los lleven a su plena autonomía; regular y evaluar los progresos y fomentar el logro de aprendizajes significativos y transferibles.

\section{PENSAMIENTO VISIBLE}

Siguiendo el planteamiento de Báez y Onrubia (2016), el pensamiento se define como un conjunto de habilidades cognitivas que pueden aprenderse y mejorarse para adaptarnos mejor al mundo en el que vivimos. Para evidenciar y apoyar el desarrollo de estas habilidades del pensamiento, Ritchhart, Church y Morrison (2014) proponen el enfoque del Visible Thinking - pensamiento visible, caracterizado por la representación observable del pensamiento.

Cuando hablamos de hacer visible el pensamiento hablamos de las estrategias y procesos de pensamiento específicos que los estudiantes utilizan para construir una comprensión más profunda. Estos son procesos que necesitan vivir en el centro de la actividad del aula y dirigir el trabajo de los profesores y los estudiantes en la medida de que los estudiantes pueden desarrollar una mayor conciencia de los procesos de pensamiento (Ritchhart et al., 2014). La necesidad de visibilizar el pensamiento en el aula radica en que "el hecho de pedir a los alumnos reiteradamente que piensen no garantiza que vayan a hacerlo de forma eficiente y vayan a convertirse en pensadores eficaces" (Swartz et al., 2017, p. 22).

Si partimos de la base de que el aprendizaje es el resultado del pensamiento (Perkins, 2008), es indudable la necesidad de la presencia del Aprendizaje Basado en el Pensamiento (ThinkingBased Learning, TBL) en la dinámica de las aulas. De ahí la necesidad de que, tal y como indica la OECD (2001), la capacidad metacognitiva sea una competencia docente. Pero, para que los docentes puedan llevar a la práctica la creación de una cultura del pensamiento, es vital que desarrollen la capacidad de cuestionarse su propio pensamiento (Figura 1).

Las rutinas del pensamiento, una de las ocho fuerzas culturas necesarias para construir culturas del pensamiento en las aulas (Ritchhart et al., 2009), son un ejemplo de estrategias 


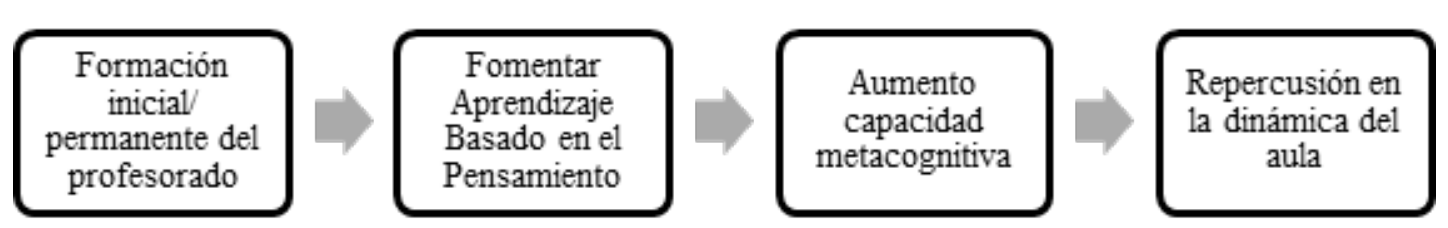

Figura 1. Metacognición en la formación docente. Fuente: elaboración propia

cognitivas de pensamiento visible. Estas pueden ser entendidas como: herramientas, estructuras y patrones de comportamiento. En primer lugar, pueden entenderse como herramientas, ya que el promueven diferentes tipos de pensamiento. En segundo lugar, pueden considerarse estructuras, puesto que se encuentran diseñadas para actuar como un andamiaje natural que permite a los alumnos alcanzar progresivos niveles de profundización en su pensamiento y, por ello, en su aprendizaje. En último lugar, pueden actuar como patrones de comportamiento, al ser estas una forma útil de pensar sobre la práctica de la enseñanza, lo cual hace a las rutinas patrones de comportamiento socialmente compartidos (Ritchhart et al., 2014).

Mediante la aplicación de las rutinas, tanto el profesorado como el alumnado podrá tomar conciencia de los procesos que nos llevan a la comprensión y al aprendizaje (Cañas, García, Pinedo y Calleja 2017). Por ejemplo, investigaciones como la de Pinedo, Acebesde Pablo, García-Martín y Cañas (en prensa), muestran como el uso de la red social Twitter

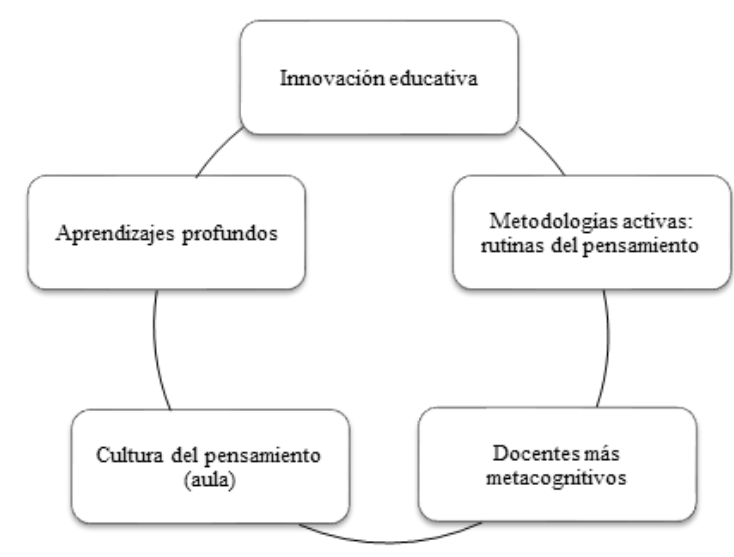

Figura 2. Innovación educativa y pensamiento. Fuente: Pinedo, García y Cañas (2017). puede fomentar la cultura del pensamiento en la formación inicial del profesorado, ya que permite contribuir al desarrollo de las habilidades relativas a la competencia aprender a aprender (Figura 2).

Precisamente, una rutina del pensamiento ampliamente utilizada para sintetizar y organizar ideas es la denominada "Generar, clasificar, conectar, elaborar-Mapas conceptuales". Autores como Richhart, Turner, y Hadar (2009) o Salmon y Lucas (2011) han recurrido a dicha rutina para visibilizar el pensamiento de los participantes de sus investigaciones.

\section{MAPAS CONCEPTUALES COMO HERRAMIEN- TA PARA HACER VISIBLE EL PENSAMIENTO}

Los mapas conceptuales son una técnica de organización gráfica que está diseñada para ayudar a los estudiantes a explorar su conocimiento y su comprensión de determinados temas (Novak y Gowin, 1984). Ritchhart, Turner y Hadar (2009) los consideraron como una herramienta metacognitiva que podía ser usada para revelar las concepciones sobre el pensamiento. Con el uso de los mapas conceptuales, estos autores buscaban diseñar una herramienta de medición que no fuera amenazante, lo suficientemente abierta para permitir respuestas ricas sobre el pensamiento que los maestros pudieran categorizar (Ritchhart et al., 2009).

Ritchhart et al. (2009) Ilevaron a cabo un estudio con 239 niños y niñas de diferentes edades que perseguía analizar sus concepciones del pensamiento. Estas concepciones se plasmaron en un mapa conceptual bajo la consigna żqué es el pensamiento?; ¿̇qué tipo de cosas podrían estar pasando realmente en tu cabeza cuando estás pensando? Esta metodología ha sido 


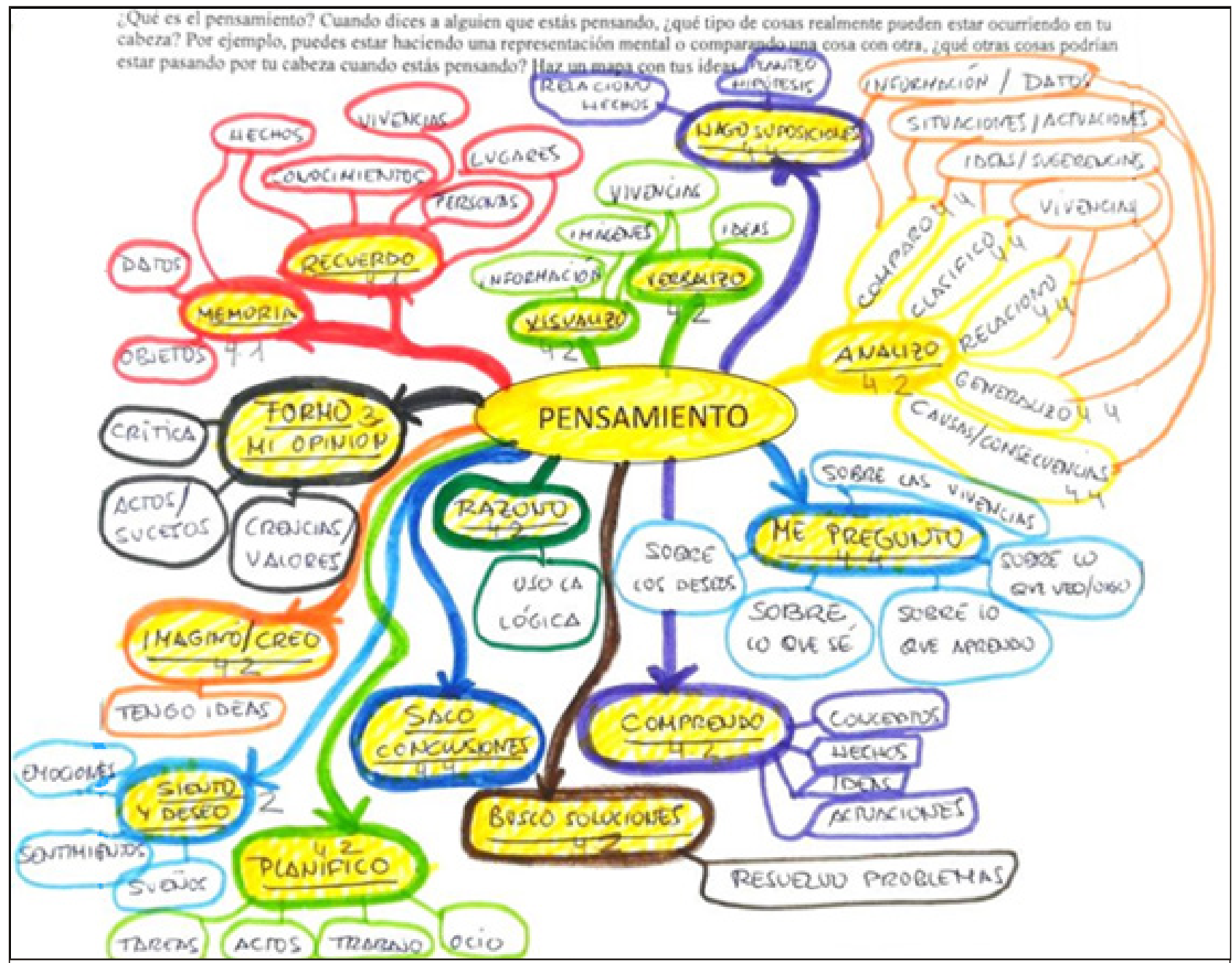

Figura 3. Ejemplo de mapa conceptual. Fuente: Elaboración propia.

utilizada en estudios posteriores por diferentes investigadores (Cañas, García, Pinedo y Calleja, 2017; Pinedo, García y Cañas, 2017; Salmon y Lucas, 2011), dando como resultado mapas conceptuales como el mostrado en la Figura 3.

\section{CATEGORIZACIÓN DEL PENSAMIENTO}

A partir de las ideas que los niños plasmaron en sus mapas conceptuales, Ritchhart et al. (2009) realizaron una categorización de sus concepciones en función de la profundidad de los procesos de pensamiento que manifestaban llevar a cabo (Tabla 1). Se pueden señalar dos grandes categorías: respuestas de tipo no estratégico, vinculadas con el aprendizaje superficial, y respuestas de tipo estratégico, relacionadas, en mayor o menor grado, con un aprendizaje profundo.

En la investigación de Ritchhart et al. (2009), a pesar de que intentaron focalizar la atención de los estudiantes hacia las estrategias del pensamiento, se encontró que en los diferentes grados las respuestas asociativas eran las que mayor porcentaje tenían, seguidas de las respuestas de tipo estratégico. Estos autores exponen que a partir de las concepciones reflejadas en los mapas conceptuales se puede proporcionar la base para una enseñanza futura que agudice las estrategias cognitivas, sin olvidar abordar la actividad emocional y el lado motivador del pensamiento.

Siguiendo esta metodología de los mapas conceptuales, estudios realizados con futuros docentes han encontrado que en torno a la mitad de las respuestas de los docentes en formación se pueden categorizar como estrategias superficiales (Pinedo, García y Cañas, 2017; Cañas, García, Pinedo y Calleja, 2017).

Por otra parte, Salmon y Lucas (2011) también se plantearon explorar y analizar las 
Tabla 1

Categorización del pensamiento.

\begin{tabular}{|c|c|c|c|}
\hline Categorías & Subcategorías & Tipos de respuestas & Ejemplos \\
\hline \multirow{3}{*}{$\begin{array}{l}\text { 1. Pensamiento } \\
\text { no estratégico }\end{array}$} & 1.1. Asociativas & $\begin{array}{c}\text { Respuestas relacionadas con el pensamiento } \\
\text { pero que no describen o identifican el acto de } \\
\text { pensar. }\end{array}$ & $\begin{array}{l}\text { Ej. Conexiones } \\
\text { neuronales, familia, } \\
\text { exámenes, ... }\end{array}$ \\
\hline & 1.2. Emocionales & $\begin{array}{l}\text { Comentarios que revelan una conexión afectiva } \\
\text { con el pensamiento (no referentes al acto de } \\
\text { pensar). }\end{array}$ & $\begin{array}{c}\text { Ej. Inseguro, alegría, difícil, } \\
\ldots\end{array}$ \\
\hline & 1.3. Meta & $\begin{array}{l}\text { Respuestas que giran en torno a la epistemología, } \\
\text { la naturaleza de la comprensión y las } \\
\text { conceptualizaciones sobre la construcción del } \\
\text { conocimiento. }\end{array}$ & $\begin{array}{c}\text { Ej. Aprender, formas ideas } \\
\text { y opiniones, } \ldots\end{array}$ \\
\hline \multirow{4}{*}{$\begin{array}{l}\text { 2. Pensamiento } \\
\text { estratégico }\end{array}$} & $\begin{array}{l}\text { 2.1. Basadas en } \\
\text { la memoria y el } \\
\text { conocimiento }\end{array}$ & $\begin{array}{l}\text { Se relacionan con el aprendizaje superficial y se } \\
\text { enfocan en el almacenamiento y recuperación } \\
\text { de la información. }\end{array}$ & $\begin{array}{c}\text { Ej. Buscar, recordar, } \\
\text { memoria, buscar en libros, } \\
\ldots\end{array}$ \\
\hline & $\begin{array}{l}\text { 2.2. Generales y no } \\
\text { específicas }\end{array}$ & $\begin{array}{l}\text { Respuestas relacionadas con la naturaleza del } \\
\text { pensamiento en general, no reflejan acciones } \\
\text { específicas que pueda tomar el pensamiento. }\end{array}$ & $\begin{array}{l}\text { Ej. Analizar, reflexionar, } \\
\text { resolución de problemas, } \\
\ldots\end{array}$ \\
\hline & $\begin{array}{l}\text { 2.3. De autorregulación y } \\
\text { motivación }\end{array}$ & $\begin{array}{c}\text { Respuestas reflejo de que el pensamiento } \\
\text { necesita ser motivado y administrado para que } \\
\text { sea más eficaz. }\end{array}$ & $\begin{array}{l}\text { Ej. Despejar la mente, } \\
\text { decirme que puedo, ... }\end{array}$ \\
\hline & $\begin{array}{l}\text { 2.4. Estrategias y } \\
\text { procesos específicos de } \\
\text { pensamiento }\end{array}$ & $\begin{array}{l}\text { Respuestas enfocadas hacia el aprendizaje } \\
\text { profundo, hacia la creación de sentido o que } \\
\text { describen un proceso de construcción de la } \\
\text { comprensión, de la resolución de problemas o de } \\
\text { la toma de decisiones. }\end{array}$ & $\begin{array}{l}\text { Ej. Considerar } \\
\text { perspectivas, hacerme } \\
\text { preguntas, sintetizar, ... }\end{array}$ \\
\hline
\end{tabular}

concepciones del pensamiento de los niños de 3-4 y 4-5 años. En este estudio no siguieron la metodología de los mapas conceptuales debido a la edad de los participantes, sino que optaron por la técnica del dibujo narrado como vehículo de expresión. Salmon (2008) sugiere que mientras se dibuja, los niños verbalizan y muestran evidencias de su actividad mental, particularmente porque el acto de dibujar compromete al niño en el uso del lenguaje, lo que genera la oportunidad para crear historias. Pero, Salmon y Lucas (2011) indican que se tiene que tener presente que el dibujo no es suficiente para hacer visible las concepciones del pensamiento del niño, sino que también es necesaria la descripción de los dibujos por parte de los niños. En ese sentido, tras indicarles a los niños la pregunta ¿̇qué es el pensamiento?, los profesores tomaron notas y se realizaron grabaciones de vídeo.

Una vez que se realizaron los dibujos, se categorizaron las respuestas de los niños en función de las categorías propuestas por Ritchhart et al. (2009). Las conclusiones de este estudio revelaron que, de nuevo, la mayor parte de las respuestas de los niños se categorizaban dentro de las respuestas asociativas, aunque en ocasiones eran capaces de comprometerse con más sofisticados tipos de pensamiento representados por las respuestas estratégicas.

En el estudio que aquí se presenta se ha llevado a cabo una réplica de la investigación realizada por Ritchhart et al. (2009), pero con profesorado en activo y en formación. El objetivo 
principal que se persigue es conocer qué concepciones sobre el pensamiento tienen estos colectivos. El hecho de conocer qué consideran los docentes que es el pensamiento puede servir para enriquecer programas de formación inicial y permanente del profesorado con estrategias que les permitan adquirir una mayor conciencia sobre qué es el pensamiento y cuáles son procesos o habilidades que lo componen. De este modo, los docentes serán conscientes de cómo se construye el pensamiento, tomarán conciencia de los recursos cognitivos implicados y podrán trabajarlos de forma sistemática en sus clases con su alumnado (Harre y Gillet, 1994).

Los objetivos específicos que vertebran esta investigación son, en primer lugar, conocer qué concepciones del pensamiento (asociativo, emocional, meta o estratégico) presenta la muestra en general, en segundo lugar, comparar las concepciones del pensamiento de los docentes en activo y los docentes en formación, y en tercer lugar, analizar qué variables pueden estar relacionadas con un pensamiento más estratégico en docentes y futuros docentes.

\section{MÉTODO}

\section{PARTICIPANTES}

Para la realización de este estudio contamos con dos muestras de análisis, una compuesta por docentes en formación (80\%) y otra compuesta por docentes en activo (20\%). La muestra total $(N=416)$ fue extraída por conveniencia en las siguientes ciudades españolas: Segovia (53,6\%), Valladolid $(42,3 \%)$ y Zaragoza $(4,1 \%)$.

La edad media de los docentes en formación es de 20,22 años (D.T=2,96), con un mínimo de 18 años y un máximo de 53 años. Un 61\% está cursando el ler curso de Grado, un 20\% de $2^{\circ}$ curso y un $19 \%$ de 3 er curso. En este grupo el $72 \%$ son mujeres, un $19 \%$ estudia el Grado de Educación Infantil, el 51\% estudia el Grado de Educación Primaria y el 30\% restante el Programa de Estudios Conjunto de Educación Infantil y Primaria (PEC).

La edad media de los docentes en activo es de 43,70 años (D.T.=9,90), con un mínimo de 22 años y un máximo de 68 años, y tienen una experiencia docente media de 20,12 años (D.T $=10,20)$, con un mínimo de 1 año y un máximo de 48 años. En este grupo el 73,8\% son mujeres, donde un $16 \%$ son docentes de Educación Infantil, un 64\% de Educación Primaria, un 4\% de Educación Secundaria y el 16\% restante tanto de Educación Infantil como de Primaria.

\section{INSTRUMENTOS}

Para la consecución de los objetivos se ha replicado el estudio realizado por Ritchhart, et al. (2009), en el cual se plantea a los participantes las siguientes preguntas: ¿̇qué es el pensamiento?; ¿̇qué tipo de cosas podrían estar pasando realmente en tu cabeza cuando estás pensando?

El instrumento utilizado para la recogida de datos fue un mapa conceptual que los participantes formaron en torno a la palabra "pensamiento" para dar respuesta a las preguntas que se les planteaban (ver Figura 3).

Los mapas conceptuales fueron debidamente analizados categorizando las respuestas tal y como se muestra en la Tabla 1 anteriormente mostrada.

\section{PROCEDIMIENTO}

La recogida de datos se ha llevado a cabo de forma progresiva. En el caso de los futuros docentes, los mapas conceptuales se efectuaron en el entorno universitario y al inicio de curso. Por su parte, la recolección de los mapas de profesorado en activo se ha llevado a cabo en congresos, jornadas y cursos de formación permanente de diversas temáticas y previo a la instrucción en la metodología del Aprendizaje Basado en el Pensamiento.

Los participantes realizaron el mapa conceptual de forma individual durante un tiempo aproximado de 5-10 minutos. Para evitar que las respuestas de los participantes se vieran condicionadas, los docentes y futuros docentes fueron informados de los términos de esta investigación tras la elaboración del mapa conceptual. De este modo, el profesorado que deseó participar en el estudio dio su consentimiento para que sus mapas 
conceptuales fueran analizados al entregárselos a los investigadores.

La confidencialidad de los datos se ha garantizado utilizando códigos de identificación en cada uno de los mapas. En definitiva, durante todo el estudio se han considerado los estándares éticos de los comités de investigación internacionales y/o nacionales, así como la declaración de Helsinki y sus enmiendas posteriores (Opazo, 2011 ).

\section{ANÁLISIS DE DATOS}

Los datos obtenidos a partir del análisis de los mapas conceptuales han sido tratados mediante programa informático de análisis cuantitativo de datos IBM SPSS Statistics Base 21.0 (IBM Corp., 2012).

En primer lugar, se han efectuado análisis descriptivos con vistas a conocer qué tipo de pensamiento predominaba en la muestra en general y en los docentes en formación y en activo en particular. Además se ha utilizado la prueba ${ }^{\prime}$ de student para muestras independientes con el fin de determinar si las diferencias entre ambos grupos eran estadísticamente significativas y se calculó el tamaño del efecto con la d de Cohen.

Por último, para conocer qué tipo de conceptualizaciones del pensamiento tienen los docentes en formación según su curso y titulación y los docentes en activo según sus años de experiencia docente y etapa educativa en la que trabajan, se han efectuado análisis descriptivos y ANOVA de un factor y se calculó el tamaño del efecto con eta cuadrado.

\section{RESULTADOS}

En primer lugar, se analizó el porcentaje medio de respuesta para cada una de las categorías de análisis en la muestra en su totalidad y se encontró que las respuestas no estratégicas suponían un $47,5 \%$ del total de las respuestas (Respuestas asociativas, Respuestas emocionales y Respuestas meta). En segundo lugar, las respuestas de pensamiento estratégico eran un 52,5\%del total (Estrategias basadas en la memoria y el conocimiento, Estrategias generales, Estrategias de autorregulación y motivación y Estrategias y procesos específicos de pensamiento).

Al analizar las subcategorías de las respuestas de pensamiento no estratégico y pensamiento estratégico (Figura 5), se puede ver que la categoría que mayor porcentaje de respuesta presenta es la de respuestas asociativas, la cual es una categoría de respuesta considerada de tipo no estratégico. Si se atiende al porcentaje total de respuestas de tipo estratégico y no estratégico se observa que está muy igualado, aunque es ligeramente superior el porcentaje de respuestas de pensamiento estratégico.

A continuación, se analizó el porcentaje medio de respuesta en cada una de las categorías de análisis en los subgrupos que componían la muestra: profesorado en formación y en activo (Figura 6 y 7 ).

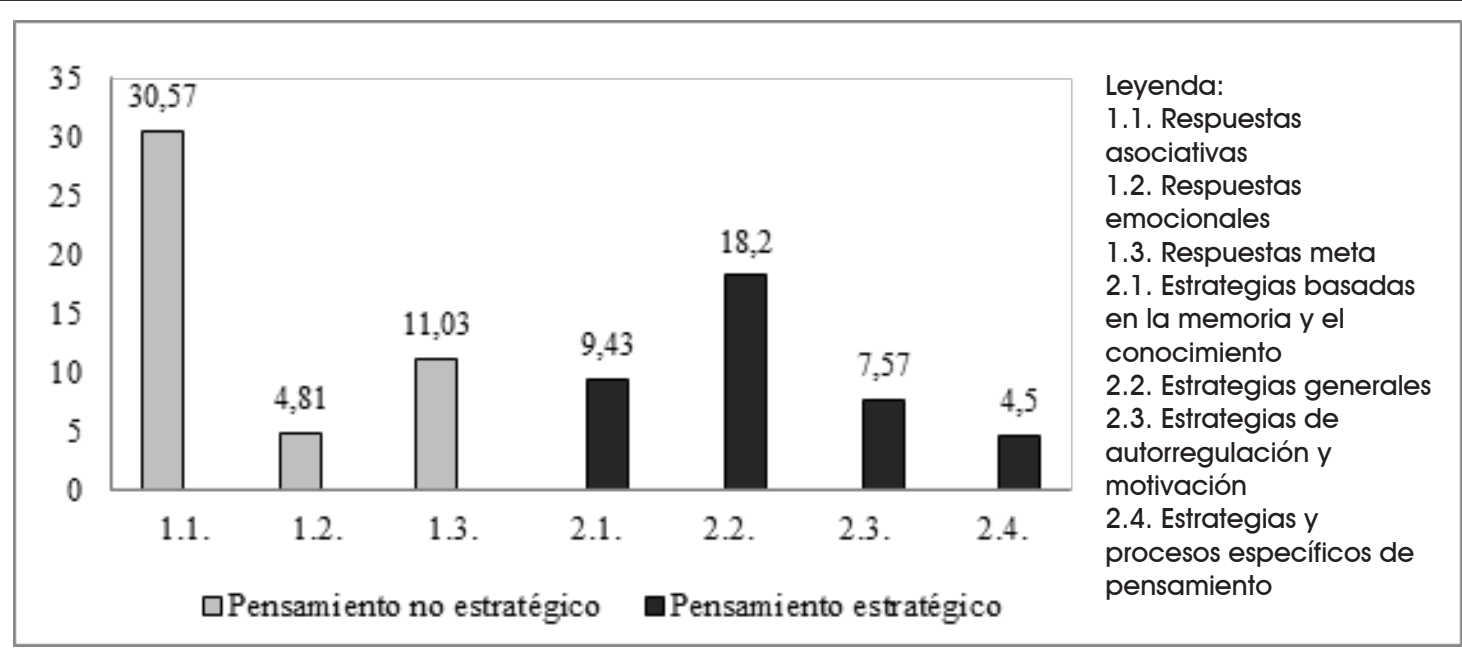

Figura 5. Porcentaje medio de respuesta para cada una de las subcategorías de pensamiento estratégico y no estratégico para la totalidad de la muestra $(N=416)$. 

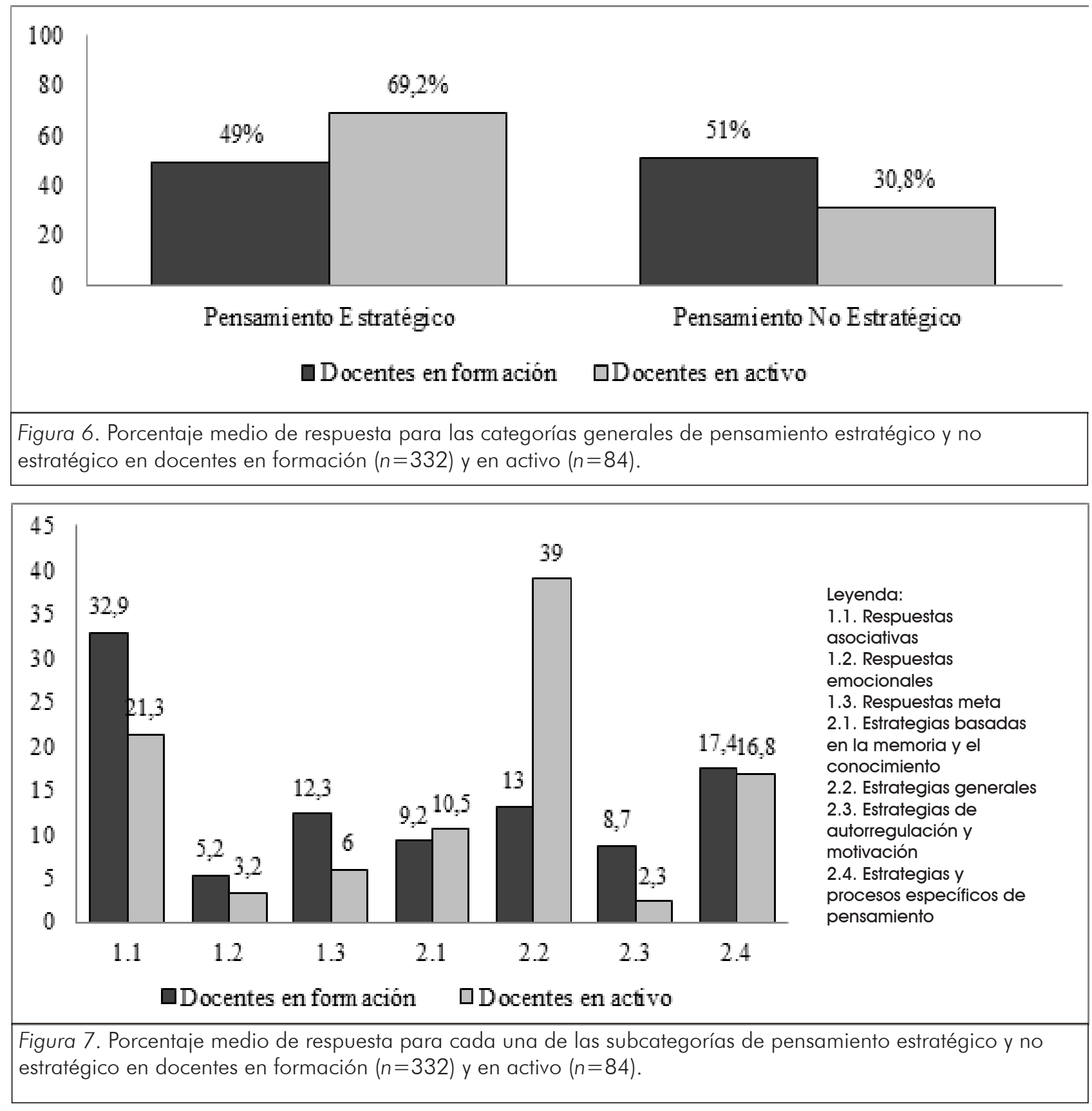

Se compararon las respuestas entre docentes en activo y docentes en formación y se obtuvieron diferencias estadísticamente significativas en las respuestas estratégicas $\left(t_{(410)}=-7.07, p=.001\right.$, $d$ de Cohen=0.82). Los docentes en activo mostraron un mayor número de respuestas estratégicas (Media $=5.06$; D.T. $=2.86$ ) que los docentes en formación (Media $=2.88$; D.T. $=2.39$ ).

Para profundizar en el análisis de cada uno de los subgrupos que compone la muestra se analizó para los docentes en formación si había diferencias entre los grupos en función del curso académico o el Grado en Educación que estaba cursando (e.g. Infantil, Primaria o PEC). Para la muestra de docentes en activo se analizó si existían diferencias en función de la experiencia docente o la etapa educativa en la que ejercían su labor docente.

\section{DATOS RELATIVOS A LOS DOCENTES EN FORMACIÓN}

Al analizar si había diferencias entre las categorías de respuesta en los docentes en formación en función del curso en el que se encontraban matriculados, se encontró que existían diferencias en las categorías generales 
$\left(F_{(2,323)}=17,89 ; p=, 001, \eta^{2}=, 10\right)$, de manera que presentaban un menor porcentaje de respuestas no estratégicas en $2^{\circ}$ curso, en comparación con $1^{\circ}(p=, 001)$ y $3^{\circ}(p=, 001)$. También se encontraron diferencias en las subcategorías de respuesta asociativas $\left(F_{(2,323)}=18,6 ; p=, 001, \eta^{2}=, 10\right)$, de estrategias basadas en la memoria y el conocimiento $\left(F_{(2,323)}=6,02 ; p=, 001, \eta^{2}=, 04\right)$, de estrategias de autorregulación y motivación $\left(\mathrm{F}_{(2,325)}=7,6\right.$; $\left.p=, 001, \eta^{2}=, 05\right)$ y de estrategias y procesos específicos de pensamiento $\left(\mathrm{F}_{(2,323)}=22,1\right.$; $\left.p=, 001, \eta^{2}=, 12\right)$. En todos los casos, las diferencias se dieron entre el $2^{\circ}$ curso con $1^{\circ}$ y $3^{\circ}$ (Tabla 2). Como se puede observar el tamaño del efecto fue especialmente importante en las diferencias en la categoría general de respuestas de pensamiento no estratégico, y en las subcategorías de respuestas asociativas y respuestas de tipo estratégico y procesos específicos de pensamiento.

Al analizar si había diferencias entre las categorías de respuesta en los docentes en formación en función del grado que cursaban, se encontró que no existían diferencias en las categorías generales, mientras que sí que mostraban diferencias, estadísticamente significativas, en las subcategorías de pensamiento estratégico denominadas estrategias generales $\left(F_{(2,326)}=5,82 ; p=, 001, \eta^{2}=, 05\right)$ y estrategias de autorregulación y motivación $\left(F_{(2,328)}=3,68\right.$; $\left.p=, 01, \eta^{2}=, 03\right)$. En las respuestas de tipo estratégico generales las diferencias se dieron entre el alumnado de Máster y el alumnado de Infantil $(p=, 01)$, Primaria $(p=, 01)$ y PEC $(p=, 002)$. Mientras que en las respuestas de autorregulación y motivación las diferencias se dieron entre el alumnado del PEC e Infantil $(p=, 03)$ (Tabla 3).

\section{DATOS RELATIVOS A LOS DOCENTES EN ACTIVO}

Al analizar si había relación entre los años de experiencia docente y las diferentes categorías de respuesta en el profesorado en activo no se encontraron diferencias significativas para ninguna de las categorías, ni generales ni subcategorías (Tabla 4).

Sin embargo, al analizar las diferencias entre las categorías de respuesta en los docentes

\section{Tabla 2}

Estadísticos descriptivos de las categorías de respuesta generales y subcategorías para los docentes en formación en función del curso.

\begin{tabular}{|c|c|c|c|c|}
\hline & & Curso & Media & D.T. \\
\hline \multirow{3}{*}{ Categorías Generales } & \multirow{3}{*}{$\begin{array}{l}\text { Respuestas de } \\
\text { pensamiento no } \\
\text { estratégico }\end{array}$} & $1^{\circ}$ & 56.6 & 34.1 \\
\hline & & $2^{\circ}$ & 28.7 & 22.7 \\
\hline & & $3^{\circ}$ & 53.5 & 35.9 \\
\hline \multirow{12}{*}{ Subcategorías } & \multirow{3}{*}{$\begin{array}{l}\text { Respuestas de tipo } \\
\text { asociativo }\end{array}$} & $1^{\circ}$ & 39.4 & 33.1 \\
\hline & & $2^{\circ}$ & 12.5 & 15.3 \\
\hline & & $3^{\circ}$ & 32.9 & 33.9 \\
\hline & \multirow{3}{*}{$\begin{array}{l}\text { Estrategias basadas } \\
\text { en la memoria y el } \\
\text { conocimiento }\end{array}$} & $1^{\circ}$ & 7.6 & 13.5 \\
\hline & & $2^{\circ}$ & 15.1 & 18.8 \\
\hline & & $3^{\circ}$ & 8.8 & 14.6 \\
\hline & \multirow{3}{*}{$\begin{array}{l}\text { Estrategias de } \\
\text { autorregulación y } \\
\text { motivación }\end{array}$} & $1^{\circ}$ & 7.7 & 15.2 \\
\hline & & $2^{\circ}$ & 15.2 & 17.3 \\
\hline & & $3^{\circ}$ & 5.7 & 11.7 \\
\hline & \multirow{3}{*}{$\begin{array}{c}\text { Estrategias y procesos } \\
\text { específicos de } \\
\text { pensamiento }\end{array}$} & $1^{\circ}$ & 12.9 & 18.7 \\
\hline & & $2^{\circ}$ & 31.2 & 19.7 \\
\hline & & $3^{\circ}$ & 17.7 & 20 \\
\hline
\end{tabular}


Tabla 3

Estadísticos descriptivos de las subcategorías de respuesta para los docentes en formación en función del grado.

\begin{tabular}{|c|c|c|c|c|}
\hline & & Grado & Media & D.T. \\
\hline \multirow{8}{*}{ Subcategorías } & \multirow{4}{*}{ Estrategias generales } & Infantil & 14.3 & 17.3 \\
\hline & & Primaria & 14.5 & 20.1 \\
\hline & & Inf+Prim & 8.8 & 15.1 \\
\hline & & Máster & 42.8 & 10.1 \\
\hline & \multirow{4}{*}{$\begin{array}{l}\text { Estrategias de } \\
\text { autorregulación y } \\
\text { motivación }\end{array}$} & Infantil & 5.66 & 11.7 \\
\hline & & Primaria & 7.7 & 13.2 \\
\hline & & Inf + Prim & 12.6 & 19.7 \\
\hline & & Máster & 0 & 0 \\
\hline
\end{tabular}

Tabla 4

Estadísticos descriptivos de las subcategorías de respuesta para los docentes en activo en función de la etapa en la que imparten clase.

\begin{tabular}{|c|c|c|c|c|}
\hline \multicolumn{2}{|c|}{} & Etapa & Media & D.T. \\
\hline \multirow{3}{*}{ Subcategorías } & $\begin{array}{c}\text { Estrategias basadas } \\
\text { en la memoria y el } \\
\text { conocimiento }\end{array}$ & Infantil & 9.28 & 11.41 \\
\cline { 3 - 5 } & & Primaria & 7.48 & 9.25 \\
\cline { 3 - 5 } & Secundaria & 26.18 & 16.76 \\
\hline
\end{tabular}

en activo en función de la etapa donde imparten docencia se encontraron diferencias estadísticamente significativas $(F(2,71)=8,29$; $\left.\mathrm{p}=, 001, \eta^{2}=, 19\right)$. Estas diferencias se daban entre los docentes que impartían docencia en Secundaria y los docentes de Infantil $(p=, 004)$, Primaria $(p=, 001)$. Los docentes de Secundaria presentaban una mayor media de respuestas categorizadas como estrategias basadas en la memoria y el conocimiento, siendo el tamaño del efecto elevado.

\section{DISCUSIÓN}

El objetivo de este estudio es analizar qué concepciones sobre el pensamiento tiene el profesorado en formación y en activo. Al examinar los datos obtenidos en la muestra general se ha encontrado que, aunque los porcentajes son similares, el porcentaje de respuestas de tipo estratégico es ligeramente superior. En este sentido, los resultados hallados en este estudio son más optimistas, ya que en estudios previos realizados con futuros docentes, algo más de la mitad de respuestas se podían categorizar como estrategias superficiales de pensamiento (Pinedo, García y Cañas, 2017; Cañas, García, Pinedo y Calleja, 2017).

A su vez, en el desglose de cada una de las categorías de respuesta, la de tipo asociativa es el más frecuente, seguida en menor porcentaje, por las categorías de pensamiento, denominadas generales y no específicas y estrategias y procedimientos específicos de pensamiento. Estos resultados son similares a los obtenidos por Ritchard et al. (2009) en su estudio sobre las concepciones del pensamiento del alumnado, por lo que podemos pensar que el tipo de pensamiento utilizado por los docentes podría ser adquirido por el alumnado.

Este aspecto sería congruente con las fuerzas culturales propuestas por Ritchhart (2015) para fomentar una cultura del pensamiento en el aula, donde el docente tiene que ser un modelo adecuado de pensamiento para facilitar que el alumnado aprenda a ser un buen pensador estratégico. Por tanto, necesitamos que el profesorado tenga concepciones del 
pensamiento de tipo estratégico para que pueda modelar en este sentido las concepciones del pensamiento de su alumnado (Harre y Gillet, 1994).

En la comparación de las concepciones del pensamiento en los docentes en activo y los docentes en formación, existen diferencias. Por un lado, los docentes en formación presentan respuestas de tipo no estratégico y estratégico en porcentajes similares, siendo este último ligeramente superior. No obstante, la subcategoría asociativa correspondiente a un pensamiento superficial es la más frecuente, siendo coincidente con estudios anteriores (Cañas, García, Pinedo y Calleja, 2017; Pinedo, García y Cañas, 2017).

Por otro lado, los docentes en activo, presentan fundamentalmente concepciones del pensamiento de tipo estratégico, en concreto del tipo general y no específico. No obstante, también recurren a la categoría asociativa, aunque en menor medida. Se puede esperar que los docentes en formación muestren una menor capacidad metacognitiva que los docentes en activo, precisamente porque aún están completando su formación, pero parece que ambos grupos presentan una excesiva presencia de respuestas no estratégicas, tal y como se advirtió en estudios previos realizados con docentes en formación (Cañas, García, Pinedo y Calleja, 2017; Pinedo, García y Cañas, 2017).

Parece que los años de formación influyen de forma positiva en la capacidad metacognitiva del profesorado, una de las competencias docentes vitales para el S.XXI que recoge la OCDE (2001), pues se ha detectado un aumento en las concepciones de pensamiento de tipo estratégico. Las diferencias entre las categorías de respuesta en los docentes en formación en función del grado que cursaban, dentro del pensamiento estratégico son estadísticamente significativas en las estrategias generales, cuyas diferencias aumentaban en los alumnos de máster en comparación a los alumnos de Infantil, Primaria y PEC. Las respuestas estratégicas de tipo autorregulación y motivación muestran diferencias principalmente entre alumnado del Educación Infantil y PEC, siendo mayores en este último grupo.
A pesar de no encontrarse diferencias significativas para ninguna de las categorías, ni generales ni subcategorías, para los docentes en activo en términos generales, sí se encuentran de forma más específica en cada una de las etapas, siendo la etapa de Secundaria la que mayor presencia tienen las concepciones de pensamiento estratégico del tipo memoria y conocimiento. Estos datos evidencian la necesidad de enriquecer la formación permanente del profesorado en materia de pensamiento (Harre y Gillet, 1994).

Las implicaciones educativas que conllevan este planteamiento docente, están directamente relacionadas con las exigencias que marcan los estándares educativos, no solo a nivel nacional, sino también por la OCDE (2001) como se mencionaba anteriormente. La propuesta de evaluar a los estudiantes en referencia a las competencias clave no es una labor sencilla para los docentes, puesto que las metodologías tradicionales no se ajustan a la adquisición de las mismas y el profesorado no tiene una formación específica en la materia (Pinedo, Caballero, Fernández, 2016). Del mismo modo, no todas las competencias plantean los mismos retos en su adquisición. En concreto, la competencia de aprender a aprender supone no solo un cambio metodológico, sino también conceptual de las prácticas educativas por parte del docente y de los estudiantes (Perkins, 2008).

Al igual que los estudios de Harre y Gillet (1994), Perkins, (2008), Ritchhart, et al. (2014), y Swartz et al. (2017), el objetivo del presente trabajo es poner en relieve la conciencia del propio proceso cognitivo para aplicar de forma más efectiva y sistemática el pensamiento eficaz del alumnado, para mejorar en su aprendizaje escolar, socioemocional y personal. Todo ello teniendo en cuenta que el fin último del sistema educativo es preparar a los alumnos para las exigencias de la sociedad actual, la sociedad del conocimiento (Coolahan, 2003; Swartz et al., 2013; Scott, 2015).

En este sentido, que los docentes y futuros docentes presenten concepciones del pensamiento superficiales y no estratégicas imposibilitará dar respuesta al objetivo marcado. 
Es por ello que el entrenamiento y formación en la metodología del Aprendizaje Basado en el Pensamiento y, en concreto, en estrategias de Visible Thinking mediante herramientas tales como las rutinas de pensamiento, posibilitará la adquisición y desarrollo de un pensamiento más estratégico (Ritchhart, et al., 2014), que permita un mejor desarrollo de las habilidades metacognitivas para un mejor funcionamiento de los procesos de pensamiento de orden superior (Ardila, 2018).

Finalmente, los beneficios educativos que suponen el empleo del pensamiento estratégico en el ámbito educativo para una adecuada adquisición de las competencias educativas y un aprendizaje significativo de las materias escolares, podrían generalizarse a otros aspectos más concretos del ámbito social y personal, como manejo de las emociones y conducta, tan necesarios para una adecuada convivencia, y habilidades de la vida diaria.

Los resultados que aquí se presentan responden a una primera fase de una investigación más amplia y de tipo longitudinal relacionada con el estudio de las concepciones del pensamiento en el profesorado y el alumnado de las diferentes etapas educativas.

Este estudio presenta como principal limitación su diseño de investigación, ya que únicamente permite describir la situación del colectivo en relación a las variables estudiadas y analizar las relaciones entre las mismas. Asimismo, la muestra no es representativa del profesorado en activo ni en formación, puesto que no tiene el tamaño adecuado ni ha sido obtenida a través de métodos de muestreo aleatorios. Aun así, este estudio permite formular algunas conclusiones interesantes y establecer propuestas futuras de investigación a través de los siguientes interrogantes ¿̇De qué forma las concepciones del pensamiento del profesorado se reflejan en las concepciones que tiene su alumnado? ¿̇La formación permanente del profesorado en relación a una enseñanza basada en el pensamiento y la comprensión impactará de forma significativa en la capacidad metacognitiva de su alumnado?

\section{- Conflicto de intereses}

Los autores declaran no tener ningún conflicto de intereses.

\section{REFERENCIAS}

Ardila, A. (2018). Is intelligence equivalent to executive functions? Psicothema, 30 (2), 159-164. http://doi.org/10.7334/ psicothema2017.329

Báez, J., y Onrubia, J. (2016). Una revisión de tres modelos para enseñar las habilidades de pensamiento en el marco escolar. Perspectiva Educacional: Formación de Profesores, 55(1), 94-113.

Bauman, Z. (2006). Modernidad líquida. Buenos Aires: Fondo de Cultura Económica. Cañas, M., García, N., Pinedo, R. y Calleja, M.A.I. (2017). ¿¿Qué concepciones tienen sobre el pensamiento los docentes en formación? En T. Ramiro-Sánchez, M.T. Ramiro y P. Bermúdez (Eds.), Libro de actas del 5th International Congress of Educational Sciences and Development (p.591). Santander: AEPC.

Coolahan, J. (2003). Attracting, developing and retaining effective teachers: contry background report for Ireland. Paris: OECD. Harre, R., y Gillet, G. (1994). The discursive mind. London: Sage Publications.

IBM Corp. (2012). IBM SPSS Statistics for Windows. Armonk, NY: IBM Corp.

Labrador, M.J., y Andreu, M.A. (2008). Metodologías activas. Valencia: Universidad Politécnica de Valencia.

Novak, J.D., \& Gowin, D. B. (1984). Learning to learn. Cambridge: Cambridge University Press.

OECD (2001). Competencies for the Knowledge Economy. In Education Policy Analysis. Paris: OCDE iLibrary. http://dx.doi.org/10.1787/ epa-2001-en

Opazo, H. (2011). Ética en investigación: desde los codigos de conducta hacia la formación del sentido ético. Revista Iberoamericana Sobre Calidad, Eficacia y Cambio En Educación, 9(2), 61-78.

Orden ECD/65/2015, de 21 de enero, por la que se describen las relaciones entre las competencias, los contenidos y los criterios 
de evaluación de la educación primaria, la educación secundaria obligatoria y el bachillerato. BOE, 25, 2015, 29, enero.

Perkins, D. (2008). La escuela inteligente. Del adiestramiento de la memoria a la educación de la mente. Barcelona: Gedisa.

Pinedo, R., Caballero, C., y Fernández, A. M. (2016). Metodologías activas y aprendizaje por competencias en las enseñanzas de grado. En J.L. Castejón (Coord.), Psicología y Educación: Presente y Futuro (pp. 448456). Alicante: ACIPE.

Pinedo, R., García, N., y Cañas, M. (2017). A study of future teachers' conceptions. En L. Gómez, A. López y I. Candel (Eds.), Proceedings of ICERI2017 Conference (pp. 4608-4612). Sevilla: IATED.

Pinedo, R., Acebes-de Pablo, A., García-Martín, N., y Cañas, M. (2018). Uso de Twitter para fomentar el pensamiento y aprendizaje en la formación inicial del profesorado. En E. García y M.J. Velasco (Eds). Herramientas Universitarias. Barcelona: Gedisa

Richhart, R., Turner, T., y Hadar, L. (2009). Uncovering students' thinking about thinking using concept maps. Metacognition Learning, 4, 145-159.
Ritchhart, R. (2015). Creating cultures of thinking. The 8 forces we must master to truly transform our schools. California: JosseyBass.

Ritchhart, R., Church, M., y Morrison, K. (2014). Hacer visible el pensamiento: cómo promover el compromiso, la comprensión y la autonomía de los estudiantes. Barcelona: Paidós.

Salmon, A. (2008). Creating a culture of thinking in the young child. Early Childhood Education Journal, 35(5), 457-461.

Salmon, A., y Lucas, T. (201 1). Exploring Young Children's Conceptions About Thinking. Journal of Research in Childhood Education, 25, 364-375.

Scott, C.L. (2015). El futuro del aprendizaje 2 ¿̇Qué tipo de aprendizaje se necesita en el siglo XXI? Investigación y Prospectiva en Educación UNESCO, París. [Documentos de Trabajo ERF, No. 14].

Swartz, R.J., Costa, A.L., Beyer, B.K., Reagan, R., y Kallick, B. (2013). El aprendizaje basado en el pensamiento. Cómo desarrollar en los alumnos las competencias del siglo XXI. Madrid: SM. 